\title{
Chave ilustrada para identificação das zoés de Brachyura do estuário da Lagoa dos Patos (RS) e região costeira adjacente
}

\author{
Rony Roberto Ramos Vieira ${ }^{1,2}$ \& Danilo Kloetz de Calazans ${ }^{1}$ \\ ${ }^{1}$ Laboratório de Crustáeos Decápodes, Instituto Oceanográfico, \\ Universidade Federal do Rio Grande - FURG, \\ Av. Itália, Km 8, CP 474, CEP 96201-900, Rio Grande, RS, Brasil \\ ${ }^{2}$ Autor para Correspondência: Rony Roberto Ramos Vieira, e-mail: ronycrab@yahoo.com
}

\begin{abstract}
VIEIRA, R.R.R. \& CALAZANS, D.K. Illustrated key for the identification of the Brachyura zoeal stages from the estuarine and nearby coastal region of the Patos Lagoon (RS). Biota Neotrop. 10(3): http://www. biotaneotropica.org.br/v10n3/en/abstract?identification-key+bn01710032010.
\end{abstract}

\begin{abstract}
The goal of this study is to provide an illustrated identification key to distinguish zoeal phase of Brachyuran larvae that occur in the estuarine and nearby coastal region of the Patos Lagoon. The key includes species, collected monthly, using a conical net ( $165 \mathrm{~cm}$ long, $60 \mathrm{~cm}$ mouth and $330 \mu \mathrm{m}$ mesh). Sampling was carried out on surface and deep waters. The identification was based on specimens reared under laboratory conditions as well as from previous published studies. This key was constructed upon external morphological characters, easy to observe under a stereomicroscope and moreover, the presentation of figures allows a non-specialist to make identifications more easily.
\end{abstract}

Keywords: Brachyura, Zoea phase, larvae, identification key.

VIEIRA, R.R.R. \& CALAZANS, D.K. Chave ilustrada para as zoés de Brachyura do estuário da Lagoa dos Patos (RS) e região costeira adjacente. Biota Neotrop. 10(3): http://www.biotaneotropica.org.br/v10n3/ pt/abstract?identification-key+bn01710032010.

Resumo: O objetivo do presente estudo é a elaboração de uma chave de identificação ilustrada das espécies de Brachyura que ocorrem no estuário da Lagoa dos Patos e sua região costeira adjacente. A chave abrange espécies as quais foram coletadas mensalmente, usando uma rede de plâncton $(165 \mathrm{~cm}$ de comprimento, $60 \mathrm{~cm}$ de boca e $330 \mu \mathrm{m}$ de malha). As amostragens foram realizadas na superfície e fundo. Os especimens foram identifcados mediante comparação com larvas cultivadas em laboratório e com descrições já existentes. A chave utiliza caracteres que não sofrem grandes transformações ao longo do desenvolvimento, e a utilização de figuras para ilustrar a chave permite a não especialistas facilmente identificar as espécies.

Palavras-chave: Brachyura, Fase zoé, larva, chave de identificação.

\section{Introdução}

O estudo da fase larval permite conhecer o ciclo de vida das espécies contribuindo para o conhecimento básico em biologia e ecologia servindo como ferramenta para avaliação do recrutamento, principalmente de espécies de interesse pesqueiro. Os estágios de zoés dos Brachyura ocorrem inteiramente na coluna d'água, livre das adaptações convergentes e divergentes sofridas pelos adultos devido aos diferentes habitats em que ocorrem e para a maioria das espécies esta fase é a principal fonte de dispersão geográfica. A constância dos caracteres nas larvas faz com que a morfologia larval represente um elemento taxonômico adicional para a identificação e classificação das espécies sendo que os primeiros trabalhos a utilizar as larvas dividiram os grupos baseados primeiramente na presença ou não de espinhos na carapaça, na morfologia do exopodito antenal e na armadura do telso (Hyman 1925, Lebour 1928, Aikawa 1929, 1933, 1937, Rice 1980, Martin 1984). Mais recentemente, Ingle (1992), Paula (1996) e Santos \& González-Gordillo (2004) realizaram trabalhos onde incluem a morfologia e o número de cerdas de outros apêndices. Para as espécies que ocorrem no litoral brasileiro somente trabalhos realizados por Fransozo et al. (1998) e Pohle et al. (1999) apresentam chave sistemática, mas somente para o primeiro estágio de zoé.
A área estuarina da Lagoa dos Patos é de $971 \mathrm{~km}^{2}$ conectandose ao Oceano Atlântico em sua porção Sul por um único canal de $20 \mathrm{~km}$ de extensão e com largura variável entre 0,5 a $3 \mathrm{~km}$. Essa área juntamente com a região costeira adjacente destaca-se entre os ambientes costeiros do Sudoeste Atlântico por sua importância ecológica e sócio-econômica (Asmus \& Tagliani 1998) servindo como berçários para larvas de várias espécies de invertebrados e vertebrados. A distribuição de populações planctônicas dentro do estuário da Lagoa dos Patos é controlada pela circulação da água doce e salgada e pela exportação entre diferentes partes do estuário. Larvas de algumas espécies de Brachyura se desenvolvem no interior do estuário, outras são transportadas para a região costeira para completar seu desenvolvimento (Vieira 2006). A correta identificação dessas larvas é de grande importância, pois permite o conhecimento sobre a dinâmica das espécies nesta região.

O objetivo desse trabalho é apresentar uma chave ilustrada para facilitar a identificação da fase zoé de Brachyura, em qualquer estágio de desenvolvimento, que ocorrem no estuário da Lagoa dos Patos e região costeira adjacente. 


\section{Material e Métodos}

A chave foi elaborada a partir de larvas cultivadas em laboratório e de larvas coletadas no plâncton e que já foram previamente descritas. As coletas foram realizadas na superfície e no fundo, com rede cônica de $165 \mathrm{~cm}$ de comprimento, $60 \mathrm{~cm}$ de diâmetro de boca e malha de $330 \mu \mathrm{m}$, com duração de 3 minutos no canal de acesso do estuário da Lagoa dos Patos e região costeira adjacente. As amostras foram preservadas em formaldeído a $4 \%$ preparados com água do mar. No laboratório as amostras foram triadas e as larvas de Brachyura separadas e identificadas.

Esta chave utiliza caracteres que não sofrem grandes transformações ao longo do desenvolvimento das zoés como por exemplo espinho lateral da carapaça, abdome, telso etc, caracteres facilmente observáveis sem a necessidade de dissecção. As cerdas dos maxilípedes nas figuras 6 e 7 foram omitidas.

A sistemática foi baseada em Ng et al. (2008).

\section{Resultados e Discussão}

Através desta chave será possível identificar as zoés em qualquer estágio de desenvolvimento.

\section{Reconhecendo uma zoé de Brachyura}

Uma zoé típica de Brachyura (exceto as zoés dos Podotremata) possui uma carapaça quase esférica (na maioria dos outros Decapoda é alongada); o rostro (espinho rostral) é dirigido para baixo e a carapaça geralmente apresenta um espinho lateral em cada lado e um espinho dorsal; o telso é em forma de furca; os apêndices torácicos são o primeiro e segundo maxilípedes e são utilizados para natação; o primeiro estágio de zoé possui olhos sésseis e o abdome possui 5 somitos; a partir do segundo estágio os olhos são pedunculados e o abdome possui 6 somitos (Figura 1) (Williamson 1974).

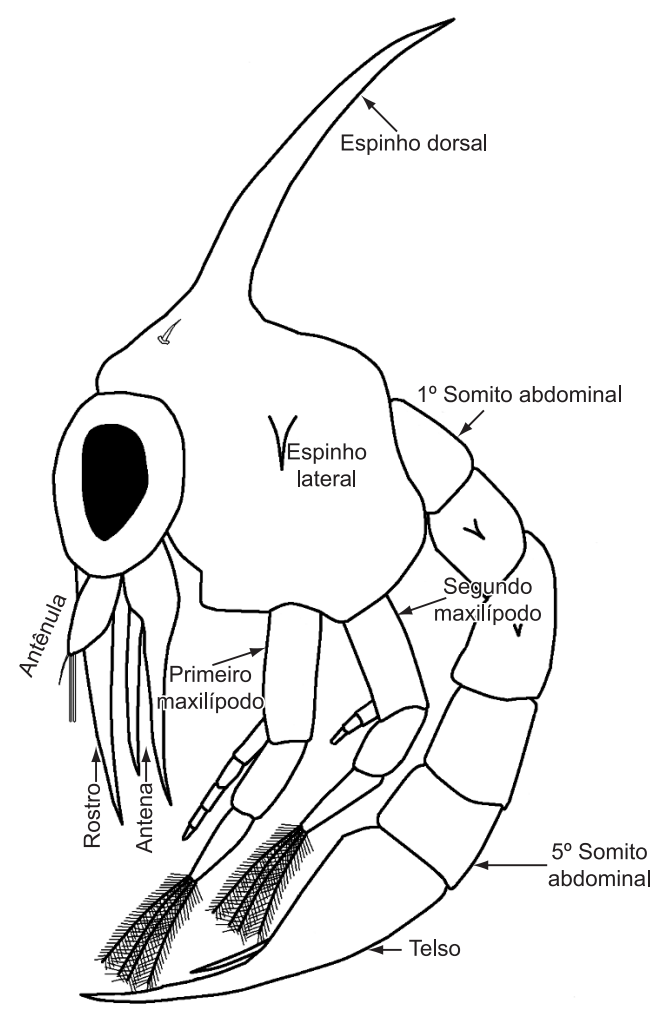

Figura 1. Vista lateral de zoé de Brachyura.

Figure 1. Lateral view of zoea of Brachyura.

\section{Número de estágios larvais}

Nos Brachyura o desenvolvimento larval pode passar totalmente dentro do ovo, eclodindo como primeiro estágio juvenil (espécies de água doce e um pequeno número de espécies marinhas) ou possuir de 1 a 13 estágios de zoés dependendo da espécie (Godbody 1960, Brossi-Garcia \& Rodrigues 1993). Os estágios larvais podem ser reconhecidos através do número de cerdas nos exopoditos dos maxilípedes sendo que no primeiro estágio tem 4 cerdas, no segundo tem 6 cerdas, no terceiro tem 8 cerdas, a partir do quinto estágio o reconhecimento ocorre pela combinação do número de cerdas nos maxilípedes com o desenvolvimento dos pleópodos (Hyman 1925).

\section{SUPERFAMÍLIA AETHROIDEA DANA, 1851}

FAMÍLIA AETHRIDAE DANA, 1851

Hepatus pudibundus (Herbst, 1785). Possui cinco estágios de zoé (Rieger \& Hebling 1993). As larvas desta espécie são encontradas nos meses de verão (Vieira 2006).

\section{SUPERFAMÍLIA LEUCOSIOIDEA SAMOUELLE, 1819}

FAMÍLIA LEUCOSIIDAE SAMOUELLE, 1819

Persephona mediterranea (Herbst, 1794). Espécie com quatro estágios de zoé (Negreiros-Fransozo et al. 1989). São encontradas principalmente no verão (Vieira 2006).

\section{SUPERFAMÍLIA MAJOIDEA SAMOULLE, 1819}

As espécies pertencentes a esta superfamília possuem dois estágios de zoé. As larvas são raras no estuário sendo encontradas mais na plataforma continental durante o ano todo (Vieira 2006).

FAMÍLIA INACHIDAE MACLEAY, 1838

Stenorhynchus seticornis (Herbst, 1788) estudada por Yang (1976).

FAMÍLIA MAJIDAE SAMOUELLE, 1819

Microphrys bicornutus (Latreille, 1825) estudada por Gore et al. (1982).

FAMÍLIA EPIALTIDAE MACLEAY, 1838.

Libinia spinosa H. Milne Edwards, 1834 estudada por Boschi \& Scelzo (1968) e Clark et al. (1998).

\section{SUPERFAMÍLIA PORTUNOIDEA RAFINESQUE, 1815}

Espécies pertencentes a esta superfamília possuem número variável de estágios entre 4 e 8 . A maior ocorrência de zoés é no verão e primavera principalmente na região costeira. A maioria das larvas capturadas no interior do estuário encontra-se no primeiro estágio de zoé e é coletada na superfície. A espécie mais abundante é Callinectes sapidus Rathbun, 1896 (Vieira 2006).

FAMÍLIA PORTUNIDAE RAFINESQUE, 1815

Arenaeus cribrarius (Lamarck, 1818) estudada por Stuck \& Truesdale (1988).

Callinectes sapidus estudada por Costlow Jr. \& Bookhout (1959).

Callinectes danae Smith, 1869 Sankarankutty et al. (1999) descreveu o primeiro estágio de zoé.

Callinectes ornatus Ordway, 1863 (D. K. Calazans, dados não publicados).

Ovalipes trimaculatus (De Haan, 1863) (D. K. Calazans, dados não publicados).

Portunus spinicarpus (Stimpson, 1871) estudada por Bookhout \& Costlow (1974) 
SUPERFAMÍLIA XANTHOIDEA MACLEAY, 1838

As espécies desta superfamília possuem quatro ou raramente cinco estágios de zoé. Ocorrem tanto dentro como fora do estuário principalmente no verão, mas também podem ser encontradas no outono e primavera (Vieira 2006).

\section{FAMÍLIA PANOPEIDAE ORTMANN, 1893}

Acantholobulus bermudensis (Benedict \& Rathbun, 1891) estudada por Martin et al. (1985).

Acantholobulus schmitti (Rathbun, 1930) estudada por Bakker et al. (1989).

Hexapanopeus angustifrons (Benedict \& Rathbun, 1891) estudada por Costlow \& Bookhout (1966).

Hexapanopeus caribbaeus (Stimpson, 1871) estudada por Vieira \& Rieger (2004).

Panopeus americanus Saussure, 1857 estudada por NegreirosFransozo (1986).

Panopeus austrobesus Williams, 1983 estudada por Montú et al. (1988).

\section{FAMÍLIA PLATYXANTHIDAE GUINOT, 1977}

Platyxanthus crenulatus A Milne Edwards, 1879 estudada por Menú-Marque (1970).

FAMÍLIA PILUMNIDAE SAMOUELLE, 1819

Pilumnus reticulatus Stimpson, 1860, estudada por Montú et al. (1989).

\section{SUPERFAMÍLIA PINNOTHEROIDEA DE HAAN, 1833}

O número de estágios larvais varia de 3 a 5 . As larvas ocorrem durante o ano todo com maiores picos no verão e primavera (Vieira 2006) e são facilmente identificáveis pela sobreposição do telso pelo quinto somito abdominal.

Na região costeira existem cinco espécies de Pinnotheridae (Martins 1996) Pinnixa brevipollex Rathbun, 1898; P.chaetopterana Stimpson, 1860; P. patagoniensis Rathbun, 1918; P. rapax Bouvier, 1917; P. sayana Stimpson, 1860, vivendo em simbiose com Callichirus major (Say, 1818) ao longo da praia do Cassino. Nenhuma das cinco espécies teve seu desenvolvimento larval estudado.

\section{SUPERFAMÍLIA OCYPODOIDEA RAFINESQUE, 1815}

FAMÍLIA OCYPODIDAE RAFINESQUE, 1815

Ocypode quadrata (Fabricius, 1787) estudada por Diaz \& Costlow (1972).

Uca uruguayensis Nobili, 1901 estudada por Rieger (1996). As larvas possuem número variável de estágios com 4,5 ou 6 zoés. As zoés são encontradas somente no verão.

\section{SUPERFAMÍLIA GRAPSOIDEA MACLEAY, 1838}

Espécies com número de estágios de zoé entre 3 e 13. As zoés das espécies Pachygrapsus gracilis (Saussure, 1858) e Pachygrapsus transversus (Gibbes, 1850) ocorrem somente no verão. Neohelice granulata (Dana, 1851) ocorre durante todo ano com maiores picos no verão. Já as outras espécies ocorrem no verão com maior abundância, outono e primavera (Vieira 2006).

FAMÍLIA GRAPSIDAE MACLEAY, 1838.

Pachygrapsus gracilis estudada por Ingle (1987) e BrossiGarcia \& Rodrigues (1993).

Pachygrapsus transversus estudada por Brossi-Garcia \& Rodrigues (1997). As zoés destas espécies são extremamente similares e é muito difícil diferenciá-las (Brossi-Garcia \& Rodrigues 1997).

FAMÍLIA SESARMIDAE DANA, 1851

Armases rubripes (Rathbun, 1897) estudada por Diaz \& Ewald (1968) e Montú et al. (1990).

FAMÍLIA VARUNIDAE MILNE EDWARDS, 1853

Neohelice granulata estudada por Boschi et al. (1967) e Cyrtograpsus angulatus Dana, 1851 estudada por Rieger \& Vieira (1997).

\section{Chave de Identificação}

1A - Antena sem exopodito (Figuras. 2a,b)

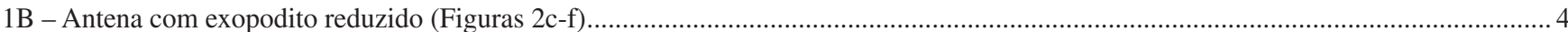

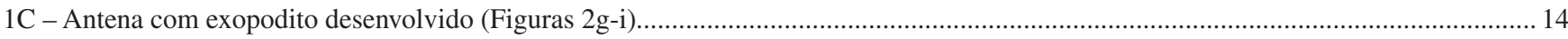

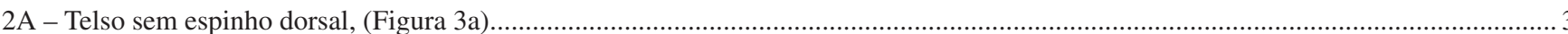

2B - Telso com espinho dorsal (Figuras 3b,c) ...............................................................................................Acantholobulus bermudensis

3A - Quinto somito abdominal maior que os demais (Figura 5a). Pinnixa spp.

3B - Quinto somito abdominal menor ou igual aos demais (Figura 5b,c) Pachygrapsus spp

4A - Telso sem espinhos lateral e dorsal (Figura 3a) Hexapanopeus angustifrons

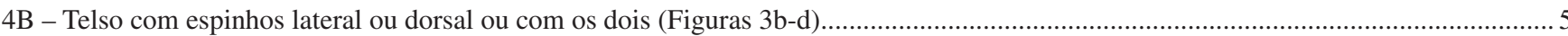

$5 \mathrm{~A}$ - Carapaça sem espinho lateral (Figuras 6a,b) Acantholobulus schmitti

5B - Carapaça com espinho lateral (Figuras 6c,d) 6

6A - Processo póstero-lateral longo no $5^{\circ}$ somito abdominal (Figura 5d)

Hexapanopeus caribbaeus

$6 \mathrm{~B}$ - Processo póstero-lateral curto no $5^{\mathrm{o}}$ somito abdominal (Figuras $5 \mathrm{~b}, \mathrm{c}$ ) 7

7A - Furca do telso com 1 espinho (Figura 3b). Panopeus americanus

7B - Furca do telso com mais de 1 espinho (Figuras 3c-d) .8 $8 \mathrm{~A}$ - Protopodito antenal com cerda no terço distal (Figura 2d). Panopeus austrobesus 
8B - Protopodito antenal com cerda na metade distal (Figuras 2e,f)

9A - Exopodito antenal com 2 cerdas (Figura 2e)

9B - Exopodito antenl com 1 espinho e 1 cerda (Figura 2f) .....

$10 \mathrm{~A}$ - Segundo espinho na furca do telso de tamanho muito reduzido (Figura 3d).

10B - Segundo espinho na furca do telso quase de mesmo tamanho do primeiro espinho (Figura 3c).

Arenaeus cribrarius

$11 \mathrm{~A}$ - Espinho lateral do $2^{\circ}$ ao $5^{\circ}$ somito abdominal pontiagudo (Figura 5e)

Callinectes sapidus

$11 \mathrm{~B}$ - Espinho lateral do $2^{\circ}$ ao $5^{\circ}$ somito não pontiagudo (Figura $5 \mathrm{f}$ )

$12 \mathrm{~A}$ - Margem posterior da carapaça com espinhos (Figura 6c)

Callinectes danae

12B - Margem posterior da carapaça sem espinhos (Figuras 6c,d).

Callinectes ornatus

13A - Ramo da furca do telso com espinhos (Figura 4a).

Portunus spinicarpus

13B - Ramo da furca do telso sem espinhos (Figuras 4b,c)....

Ovalipes trimaculatus

14A - Carapaça sem espinho lateral (Figuras 6a,b)

14B - Carapaça com espinho lateral (Figuras 6c,d)

$15 \mathrm{~A}$ - Telso com 1 par de cerdas lisas na região mediana (Figura 4b)

Uca uruguayensis

15B - Telso sem cerdas lisas na região mediana (Figura 4c)

16A - Carapaça sem espinho rostral (Figuras 6a,b)

Stenorhynchus seticornis

16B - Carapaça com espinho rostral (Figuras 6c,d)

17A - Espinho rostral menor que o protopodito antenal (Figura 7a)

13B - Espinho rostral igual ao tamanho do protopodito (Figura 6d)

Armases rubripes

$18 \mathrm{~A}$ - Protopodito antenal sem cerdas (Figuras 2a-c)

Libinia spinosa

18B - Protopodito antenal com cerdas (Figuras 2d-i)

Microphrys bicornutus

19A - Furca do telso com espinhos (Figuras 3b-d)

19B - Furca do telso sem espinhos (Figura 3a)

20A - Furca do telso com 1 espinho (Figura 3b).

.Platyxanthus crenulatus

20B - Furca do telso com mais de 1 espinho (Figuras 3c,d)

21

21A - Telso com 1 espinho lateral e 1 dorsal (Figura 4c)

Hepatus pudibundus

21B - Telso com 2 espinhos laterais e 1 dorsal (Figuras 3c,d)

Pilumnus reticulatus

$22 \mathrm{~A}$ - Carapaça com protuberância na região frontal (Figuras $6 \mathrm{c}$ e $7 \mathrm{~b}$ )

Ocypode quadrata

22B - Carapaça sem protuberância na região frontal (Figura 6d)

$23 \mathrm{~A}$ - Exopodito antenal com 1 cerda na região mediana (Figura $2 \mathrm{~h}$ ).

Neohelice granulata

23B - Exopodito antenal com 2 cerdas na região mediana (Figura 2e )

..Cyrtograpsus angulatus
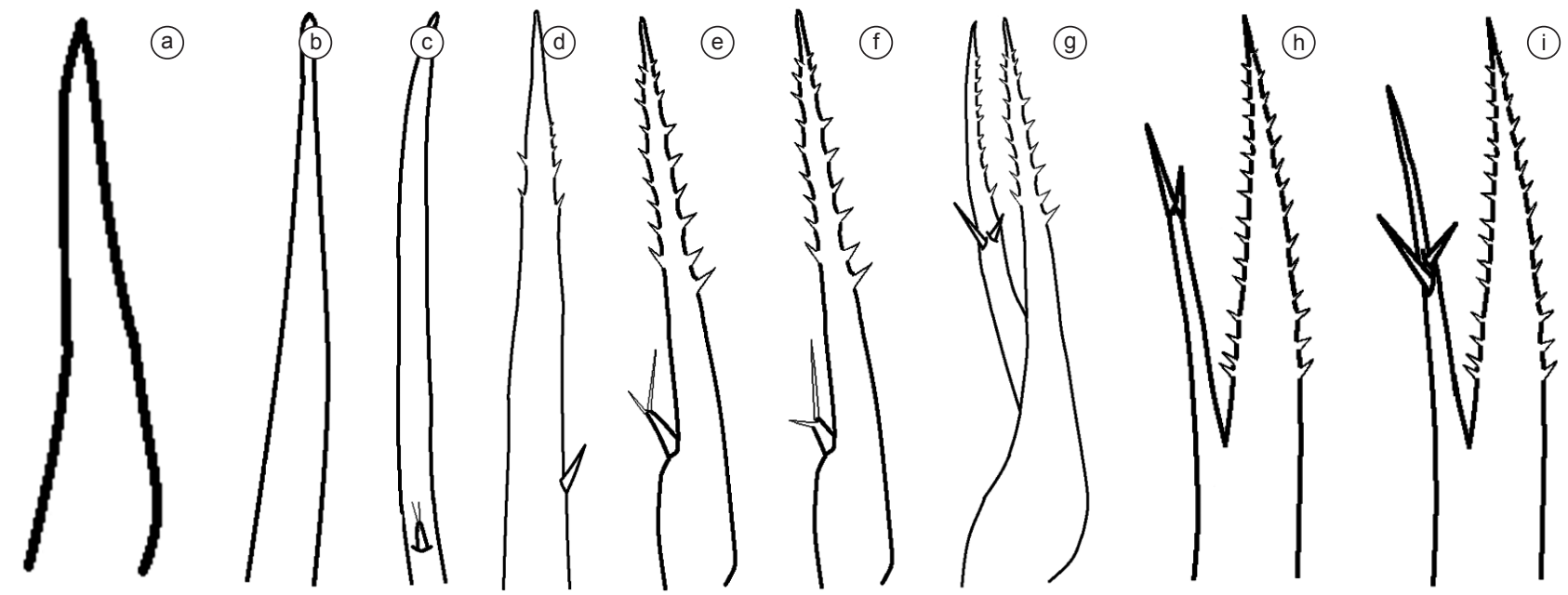

Figura 2. Antena de zoé de Brachyura.

Figure 2. Antenna of zoea of Brachyura. 


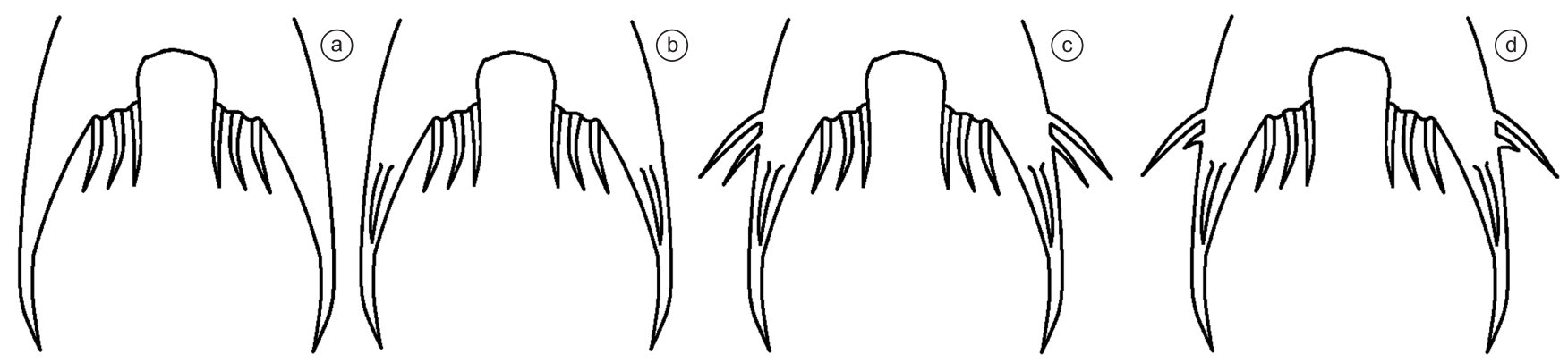

Figura 3. Vista dorsal de telso de zoé de Brachyura.

Figure 3. Dorsal view of telson of zoea of Brachyura.
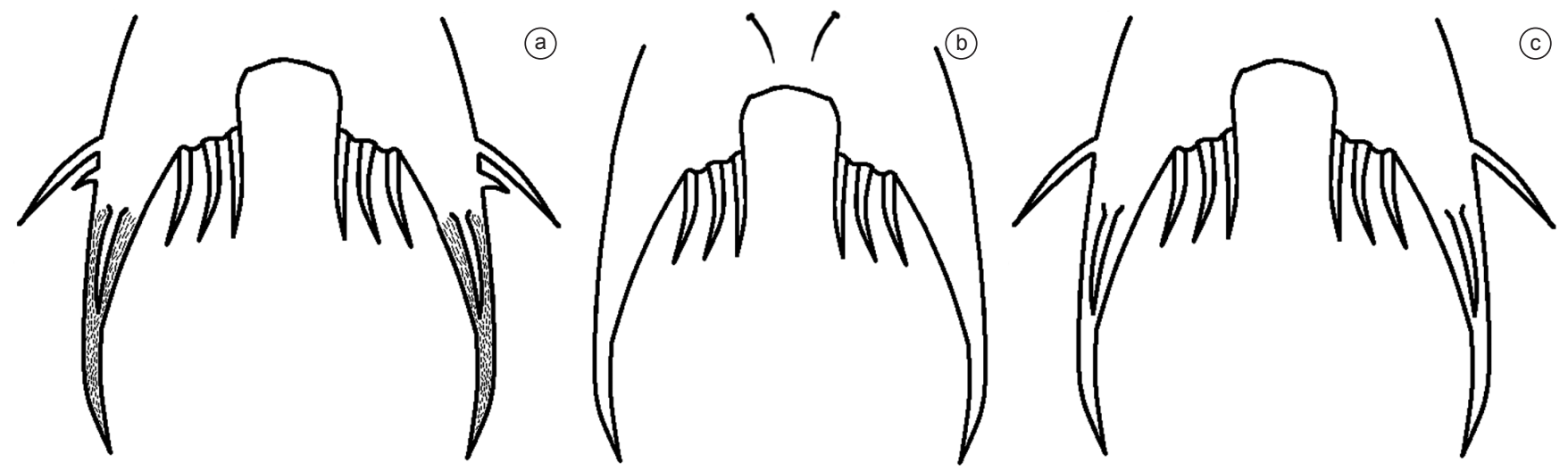

Figura 4. Vista dorsal de telso de zoé de Brachyura.

Figure 4. Dorsal view of telson of zoea of Brachyura.
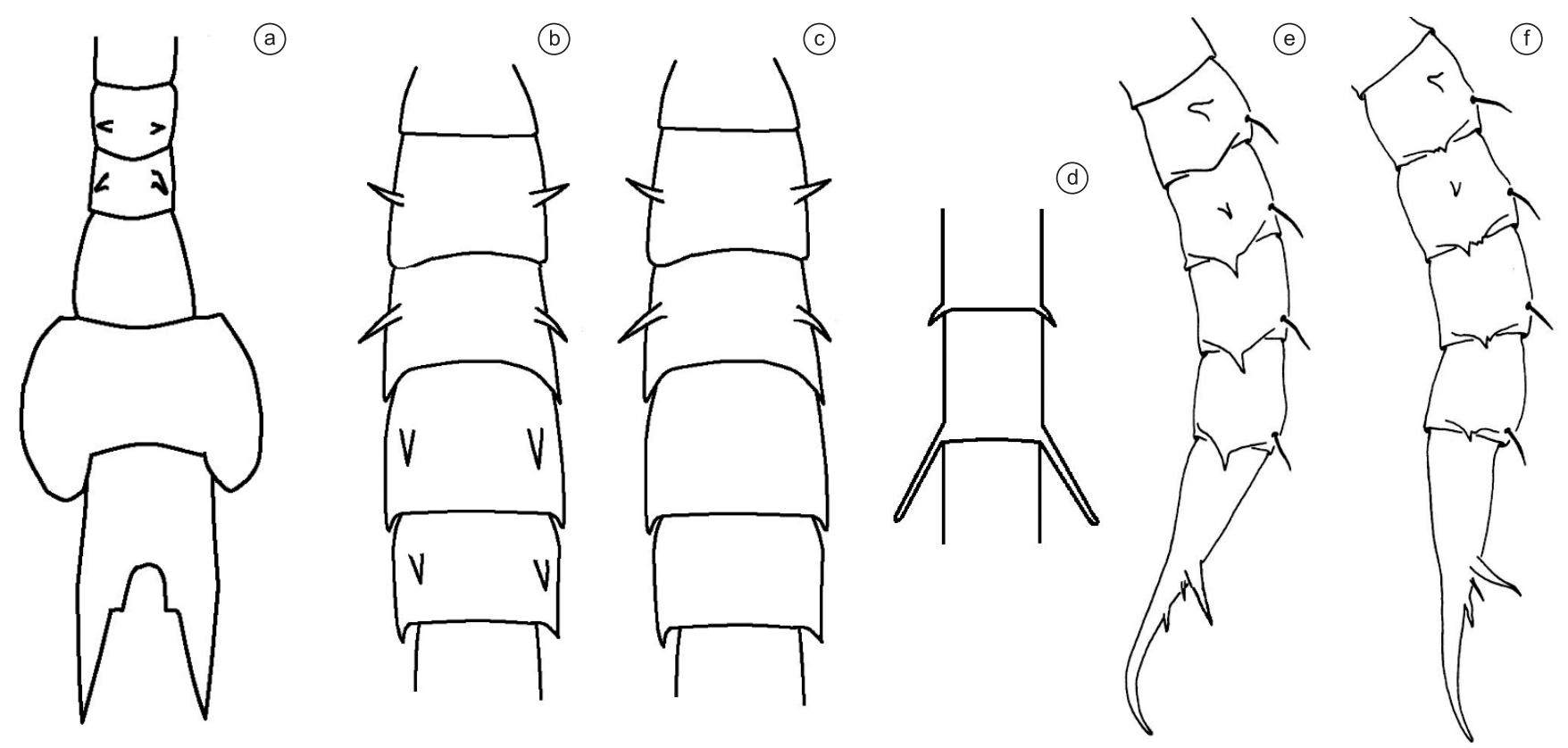

Figura 5. Abdome de zoé de Brachyura. a-d) vista dorsal e e,f) vista lateral.

Figure 5. Abdomen of Zoea of Brachyura. a-d) dorsal view e e,f) lateral view. 

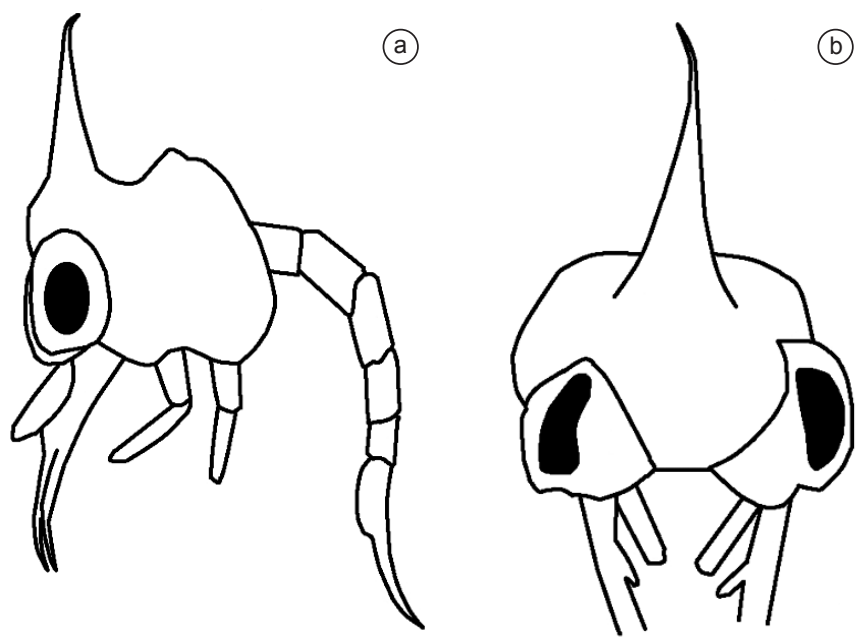

Figura 6. a, c, d), Vista lateral; b) vista frontal de zoé de Brachyura.

Figure 6. a, c, d) lateral view; b) frontal view of zoea of Brachyura.

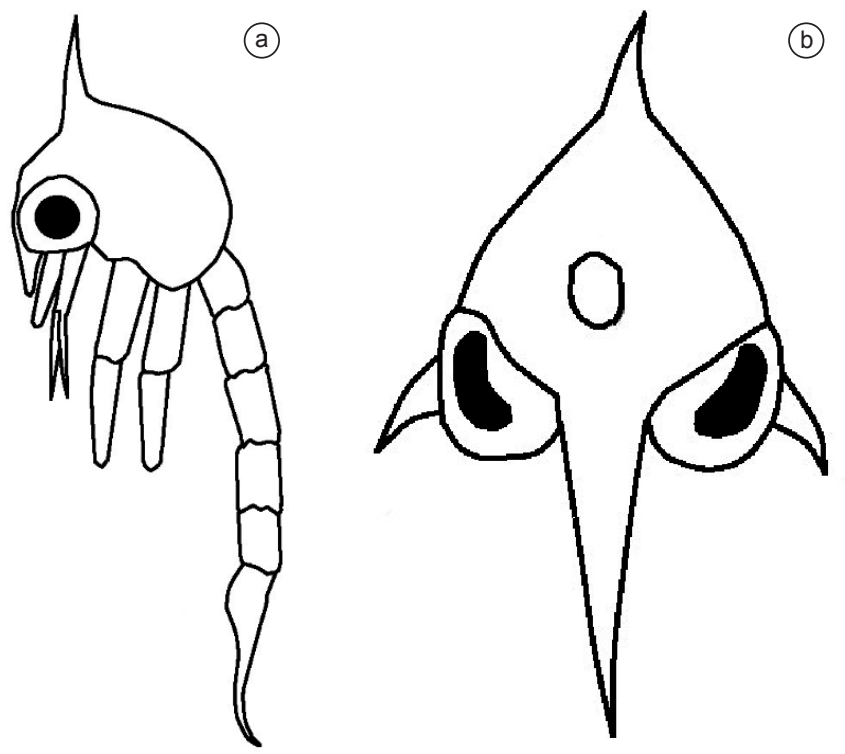

Figura 7. a) vista lateral; b) vista frontal de zoé de Brachyura.

Figure 7. a) lateral view; b) frontal view of zoea of Brachyura.

\section{Referências Bibliográficas}

AIKAWA, H. 1929. On larval forms of some Brachyura. Rec. Oceanogr. Works Jp. 2(1):17-55.

AIKAWA, H. 1933. On larval forms of some Brachyura, Paper II: A note on inderteminable zoeas. Rec. Oceanogr. Works Jp. 5:124-254.

AIKAWA, H. 1937. Further notes on Brachyuran larvae. Rec. Oceanogr. Works Jp. 9(1):87-162.

ASMUS, M.L. \& TAGLIANI, P.R.A. 1998. Considerações sobre o manejo ambiental. In Os Ecossistemas Costeiro e Marinho do Extremo Sul do Brasil. (U. Seeliger, C. Odebrecht \& J.P. Castelo, eds.). Ecoscientia, Rio Grande, p. 227-229.

BAKKER, C., MONTÚ, M., ANGER K. \& FERNANDES, L.L. 1989. Larval development of Hexapanopeus schmitti Rathbun, 1930 (Decapoda, Brachyura, Xanthidae) reared in the laboratory. Neritica. 4(1/2):137-164.

BOOKHOUT, C.G. \& COSTLOW JR, J.D. 1974. Larval development of Portunus spinicarpus reared in the laboratory. Bull. Mar. Sci. 24(1):20-51.
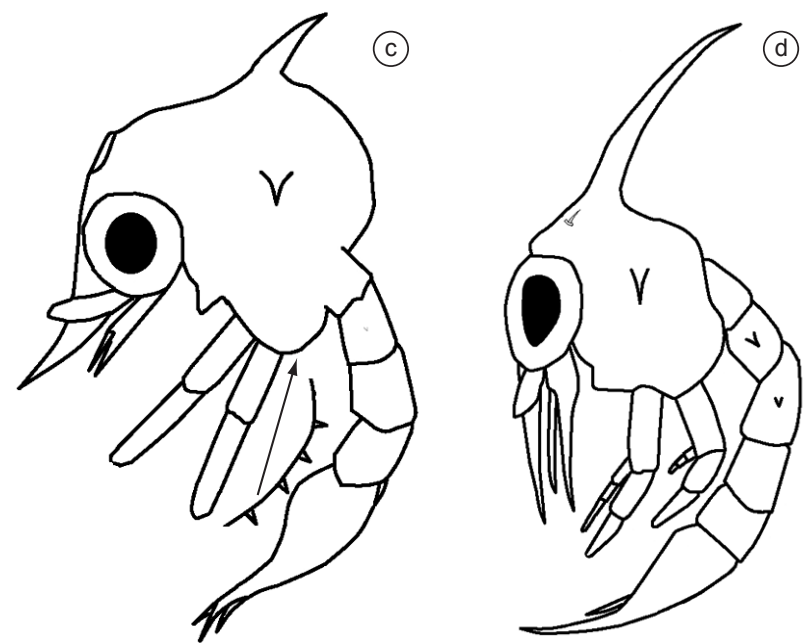

BOSCHI, E.E. \& SCELZO, M.A. 1968. Larval development of the spider crab Libinia spinosa $\mathrm{H}$. Milne Edwards, reared in the laboratory (Brachyura, Majidae). Crustaceana suppl. 2:170-180.

BOSCHI, E.E., SCELZO, M.A \& GOLDSTEIN, B. 1967. Desarrollo larval de dos especies de Crustaceos Decapodos en el laboratorio, Pachycheles haigae Rodrigues da Costa (Porcellanidae) y Chasmagnathus granulata Dana (Grapsidae). Bol. Inst. Biol. Mar del Plata. 12:4-46.

BROSSI-GARCIA, A.L. \& RODRIGUES, M.D. 1993. Zoeal morphology of Pachygrapsus gracilis (Saussure, 1858) (Decapoda, Grapsidae) reared int he laboratory. Invertebr. Reprod. Dev. 24(3):197-204.

BROSSI-GARCIA, A.L. \& RODRIGUES, M.D. 1997. Zoeal morphology of Pachygrapsus transversus (Gibbes) (Decapoda, Grapsidae) reared in the laboratory. Rev. Bras. Zool. 14(4):803-819.

CLARK, P.F., CALAZANS, D.K. \& RODRIGUES, S.S. 1998. Libinia spinosa H. Milne Edwards, 1834 (Crustacea: Majidae: Pisinae): a reappraisal of larval characters from laboratory reared material. Invertebr. Reprod. Dev. 33(2-3):145-157.

COSTLOW Jr., J.D. \& BOOKHOUT, C.G. 1959. The larval development of Callinectes sapidus Rathbun, reared in the laboratory. Biol. Bull. 116(3):373-396.

COSTLOW Jr., J.D. \& BOOKHOUT, C.G. 1966. Larval development of the crab Hexapanopeus angustifrons. Chesap. Sci. 7(3):148-156.

DIAZ, H. \& COSTLOW, J.D. 1972. Larval development of Ocypode quadrata (Brachyura: Crustacea) under laboratory conditions. Mar. Biol. 15(2):120-131.

DIAZ, H. \& EWALD, J.J. 1968. A comparison of the larval development of the Metasesarma rubripes (Rathbun) and Sesarma ricordi H. Milne Edwards (Brachyura, Grapsidae) reared under laboratory conditions. Crustaceana Sup. 11:225-248.

FRANSOZO, A., CUESTA, J.A. \& NEGREIROS-FRANSOZO, M.L. 1998. The first zoeal stage of two species of Grapsidae (Decapoda, Brachyura) and a key to such larvae from the Brazilian coast. Crustaceana 71(3):331343.

GODBODY, I. 1960. Abbreviated development in a pinnotherid crab. Nature, Lond, 185:704-705.

GORE, R.H., SCOTTO, L.E. \& YANG, W.T. 1982. Microphrys bicornutus (Latreille, 1825): the complete larval development under laboratory conditions with notes on other Mithracinae larvae (Decapoda: Brachyura: Majidae). J. Crust. Biol. 2(4):514-534.

HYMAN, O.W. 1925. Studies on the larvae of crabs of the family Xanthidae. Proc. U.S. Nat. Mus. 67(3):1-22. 
INGLE, R. 1987. The first zoea of three Pachygrapsus and of Cataleptodius floridanus (Gibbes) from Bermuda and Mediterranean (Crustacea: Decapoda: Brachyura). Bull. Br. (Nat. Hist.) Zool. 52(1):31-41.

INGLE, R. 1992. Larval stages of northeastern atlantic crab. Chapman Hall, London.

LEBOUR, M.V. 1928. The larval stages of the Plymouth Brachyura. Proc. Zool. Soc. London 473-560.

MARTIN, J.W. 1984. Notes and bibliography on the larvae of xanthid crabs, with a key the known xanthid zoeas of the western atlantic and gulf of Mexico. Bull. Mar. Sci. 34(2):220-239.

MARTIN, J.W., TRUESDALE, F.M. \& FELDER, D.L. 1985. Larval development of Panopeus bermudensis Benedict and Rathbun, 1891 (Brachyura, Xanthidae) with notes on zoeal characters in xanthid crabs. J. Crust. Biol. 5(1):84-105.

MARTINS, S.T.S. 1996. Os pinnotheridae de Santa Catarina e Rio Grande do Sul, Brasil (Decapoda, Brachyura). Rev. Bras. Zool. 13(1):1-26.

MENÚ-MARQUE, S. 1970. Desarrollo larval del cangrejo Platyxanthus crenulatus (A. Milne Edwards, 1879) en el laboratório (Decapoda, Brachyura, Xanthidae). Physis 29(9):477-494.

MONTÚ, M., ANGER, K, BAKKER, C., ANGER, V. \& FERNANDES, L.L. 1988. Larval development of the brazilian mud crab Panopeus austrobesus Williams, 1983 (Decapoda: Xanthidae) reared in the laboratory. J. Crust. Biol. 8(4):594-613.

MONTÚ, M., ANGER, K. \& BAKKER, C. 1990. Variability in the larval development of Metasesarma rubripes (Decapoda, Grapsidae) reared in the laboratory. Neritica. (1):113-128.

MONTÚ, M., BAKKER, C. \& FERNANDES, L.L. 1989. Desarrollo larval de Pilumnus reticulatus Stimpson, 1860 (Decapoda, Brachyura, Xanthidae) criada en laboratorio. Neritica, Pontal do Sul PR. 4(1/2):165-190.

NEGREIROS-FRANSOZO, M.L. 1986. Desenvolvimento pós-embrionario de Panopeus americanus Saussure, 1857 (Decapoda, Xanthidae), em laboratório. Rev. Brasil. Biol. 46(1):173-188.

NEGREIROS-FRANSOZO, M.L., FRANSOZO, A. \& HEBLING, N.J. 1989. Larval development of Persephona mediterranea (Herbst, 1794) (Brachyura, Leucosiidae) under laboratory conditions. Crustaceana 57(2):177-193.

NG, P.N., GUINOT, D. \& DAVIE, P.J.F. 2008. Systema Brachyurorum: Part I. An Annotated checklist of Extant Brachyuran crabs of the world. Raffles Bull. Zool. 17:1-286.

PAULA, J. 1996. A key and bibliography for the identification of zoeal stages of brachyuran crabs (Crustacea, Decapoda, Brachyura) from the Atlantic coast of Europe. J. Plankton Res. 18(1):17-27.
POHLE. G., MANTELATTO, F.L.M., NEGREIROS-FRANSOZO, M.L. \& FRANSOZO, A. 1999. Larval decapoda (Brachyura). In South Atlantic Zooplankton (D. Boltovskoy, ed.). p. 1281-1351.

RICE, A.L. 1980. Crab zoeal morphology and its bearing on the classification of the Brachyura. Trans. Zool. Soc. Lond. 35:271-424.

RIEGER, P.J. \& HEBLING, N.J. 1993. Desenvolvimento larval de Hepatus pudibundus (Herbst, 1785) (Decapoda, Calappidae), em laboratório. Rev. Brasil. Biol. 53(4):513-528.

RIEGER, P.J. \& VIEIRA, R.R.R. 1997. Desenvolvimento larval de Cyrtograpsus angulatus Dana (Crustacea, Decapoda, Grapsidae) em laboratório. Revta. Bras. Zool. 3:601-623.

RIEGER, P.J. 1996. Desenvolvimento larval de Uca (Celuca) uruguayensis Nobili, 1901 (Crustacea, Decapoda, Ocypodidae), em laboratório. Nauplius. 4:73-103.

SANKARANKUTTY, C., HONG, S.Y \& KIM, K.B. 1999. Description of laboratory reared first zoea of Callinectes danae Smith (Crustacea, Decapoda, Portunidae). Rev. Bras. Zool. 16(Supl. 2):45-49.

SANTOS, A. \& GONZÁLEZ-GORDILLO, J.I. 2004. Illustrated keys for the identication of the Pleocyemata (Crustacea: Decapoda) zoeal stages, from the coastal region of south-western Europe. J. Mar. Biol. Ass. UK. 84:205-227.

STUCK, K.C. \& TRUESDALE, F.M. 1988. Larval development of the speckled swimming crab, Arenaeus cribrarius (Decapoda: Brachyura: Portunidae) reared in the laboratory. Bull. Mar. Sci. 42(1):101-132.

VIEIRA, R.R.R. \& RIEGER, P. J. 2004. Larval development of Hexapanopeus caribbaeus (Stimpson, 1871) (Crustacea, Decapoda, Xanthoidea, Panopeidae) reared under laboratory conditions. J. Plank. Res. 26(10):1175-1182.

VIEIRA, R.R.R. 2006. Identificação, abundância e deistribuição das fases larvais das espécies de Brachyura (Crustacea, Decapoda) no estuário da Lagoa dos Patos e região costeira adjacente. Tese de doutorado, Universidade Federal do Rio Grande - FURG, Rio Grande - RS.

WILLIAMSON, D.I. 1974. Larval characters and the origin of crabs (Crustacea, Decapoda, Brachyura). Thalassia Jugos. 10(1/2):401-414.

YANG, W.T. 1976. Studies on the western Atlantic arrow crab genus Stenorhynchus (Decapoda, Brachyura, Majidae). I. Larval characters of two species and comparison with other larvae of Inachinae. Crustaceana 31(2):157-177. 